The concept and challenges of TomoTherapy accelerators

This article has been downloaded from IOPscience. Please scroll down to see the full text article.

2011 Rep. Prog. Phys. 74086701

(http://iopscience.iop.org/0034-4885/74/8/086701)

View the table of contents for this issue, or go to the journal homepage for more

Download details:

IP Address: 155.105.7.44

The article was downloaded on 14/09/2011 at 09:08

Please note that terms and conditions apply. 


\title{
The concept and challenges of TomoTherapy accelerators
}

\section{Claude J Bailat, Sébastien Baechler, Raphael Moeckli, Marc Pachoud, Olivier Pisaturo and François $O$ Bochud}

Institute of Radiation Physics, Grand-Pré 1, CH-1007 Lausanne, Switzerland

E-mail: claude.bailat@chuv.ch

Received 22 March 2011, in final form 24 June 2011

Published 28 July 2011

Online at stacks.iop.org/RoPP/74/086701

\begin{abstract}
A currently used intensity-modulated radiotherapy system is the TomoTherapy® Hi-Art巴 accelerator (Tomotherapy Inc., Madison, WI, USA), which started clinical treatments at the beginning of the new millennium. The innovative idea behind tomotherapy units is to marry an $\mathrm{x}$-ray computed tomography unit with a linear particle accelerator. This concept has answered some of the needs of the medical physicist community, but epidemiological evaluations are still needed in order to compare the technique with other modalities. This paper summarizes the basic concepts of tomotherapy units as well as current challenges and implications for users.

(Some figures in this article are in colour only in the electronic version)
\end{abstract}

This article was invited by G Gillies.

\section{Contents}

1. Introduction

2. Particularities of tomotherapy

2.1. Design characteristics

2.2. Beam characteristics

2.3. Delivery characteristics

2.4. Imaging characteristics

2.5. Treatment planning
2.6. Dose distribution

2.7. Commissioning and $Q A$

2.8. Radiation protection

2.9. Dosimetry

3. Future opportunities

4. Conclusions

References

\section{List of acronyms}

AAPM

CPE

CPU

CBCT

CT

DVH

EPID

FAT

GEM

GPU

IAEA

IGRT

IMAT
American Association of Physicists in Medicine charged particle equilibrium central processing unit cone-beam x-ray computed tomography $\mathrm{x}$-ray computed tomography dose volume histogram electronic portal imaging devices fluence attenuation table General Electrics Medical graphic processing unit International Atomic Energy Agency Image-guided radiation therapy intensity-modulated arc therapy system
IMRT

IVDT

LINAC

$\mathrm{MC}$

MLC

MOSFET metal-oxide-semiconductor field-effect transistor

MVCT megavolt x-ray computed tomography

NCRP National Council on Radiation Protection and Measurements

NIST National Institute of Standards and Technology

NPL National Physical Laboratory

NVBB non-voxel broad-beam

QA quality assurance

SAD source-to-axis distance 
SBRT stereotactic body radiotherapy

TERMA total energy released per unit of mass

TG

TPS Task Group

TRS Technical Report Series treatment planning system

VMAT volumetric modulated arc therapy

\section{Introduction}

This paper focuses on TomoTherapy® Hi-Art $₫$ (Tomotherapy Inc., Madison, WI, USA) accelerators, referred to hereinafter simply as tomotherapy units, and their inherent challenges for medical physicists. Furthermore, we do not want to go into the details of the history of radiotherapy in general, especially when standard medical physics textbooks and comprehensive review papers are plentiful, e.g. Karzmark (1984), Meredith (1984), Van Dyk (1999), Thwaites and Tuohy (2006), S Balter and J Balter (2008), Hannoun-Levi et al (2010). However, we will provide a brief historical overview in order to put tomotherapy into its historical context. This paper is not intended for radiotherapy experts and will not go deeply into technical details, but we do intend the references to offer many points of content expansion for keen readers.

Radiotherapy is an essential element in the treatment of cancer patients; it is used alone or in combination with surgery or chemotherapy, and it has become a well-recognized curative and palliative therapy. Because of localization uncertainties, a margin of normal tissue around the tumour is included in the treatment region. These uncertainties can be caused by natural movements, such as respiration or bladder filling, or by displacement of the tumour in relationship to external markers. The holy grail of radiotherapy is to be able to deliver a lethal dose of radiation to tumours while simultaneously preserving normal tissue, thus radically eliminating cancer cells with the lowest possible treatment toxicity, which implies minimizing margins.

In radiotherapy, the total treatment dose is delivered over time in fractions. This concept was developed out of necessity, because of limitations of the first radiotherapy units. Nowadays, biological reasons justify fractionation. Indeed, tumour cells have a typically less efficient repair mechanism, which allows healthy cells to recover relatively better between fractions. Furthermore, tumour cells in a radio-resistant phase are expected to enter a more radio-sensitive phase for the next fraction.

Historically, the last decade of the 19th century is where radiotherapy has its origins. In 1895, W C Röntgen discovered X-rays, and one year later, $\mathrm{H}$ Becquerel reported on natural radioactivity. In 1898, Pierre and Marie Curie discovered radium. Shortly after these discoveries, therapy using radiation was already proposed. In February 1896 in Chicago, E H Grubbe founded the first radiation therapy facility. Anecdotally, he died in 1960 at the respectable age of 85 , and is reported as the first person to have been injured by radiation.

Early on in the evolution of radiotherapy, the advantages of higher energy radiation were recognized. However, technical barriers did not permit megavoltage accelerators until the 1930s. Historical accounts see this period as the passage of radiotherapy from palliative to curative. Indeed, the following decades saw radiotherapy moving from a qualitative technique performed by a handful of talented experts to a quantitative therapy allowing treatment to be carried out on a larger scale.

The 1950s saw the first linear particle accelerators (LINACs) dedicated to radiotherapy operational in England and the United States. The evolution thereafter was remarkable, in the 1960s less than 20 LINACs were in use in few privileged countries; in the 1980s nearly 80 were running and nowadays there are thousands worldwide. The LINAC became simultaneously the radiotherapy instrument of choice over van de Graafs, betatrons and Co-60 irradiators. Its beginnings were modest and technological advances have permitted the LINAC to impose itself since the 1970s with an overall design which is still currently in use.

In the 1970s, the invention and subsequent medical use of the $\mathrm{x}$-ray computed tomography $(\mathrm{CT})$ led to 2D radiotherapy, and 20 years later, advances in computer technology made $3 \mathrm{D}$ conformal radiotherapy possible. Currently, image-guided radiation therapy (IGRT) refers to many techniques, which apply various types of imaging in order to control patient positioning (Huq et al 2008, Verellen et al 2008, Alongi and Di Muzio 2009, Korreman et al 2010).

Finally, in the 2000s, intensity-modulated radiotherapy (IMRT) became a major technology. In the future, the clinical introduction of imaging techniques with better soft tissue contrast or functional capacities, such as magnetic resonance imaging and positron emission tomography, is the logical step for IGRT systems. Recently, the technology of integrating imaging techniques, such as cone-beam-CT (CBCT), has reached clinical application. Currently in IMRT and radiotherapy in general, much effort is being invested to improve the conformity of dose distribution as well as using tumour tracking schemes.

Before the acceptance and widespread use of IMRT, in the late 1980s, the technique that would become known as tomotherapy was being shaped at the University of Wisconsin. A thorough history of the birth and early years of the technique was written by one of its designers, Mackie (2006), and some milestones are summarized in figure 1 . The very first paper on tomotherapy was published in 1993 (Mackie et al 1993). The development of tomotherapy at the physical laboratory of the University of Wisconsin was supported through federal funding and General Electric Medical Systems until 1997. In 1997, under financial pressure, TomoTherapy Inc. was founded to pursue the development of the clinical helical tomotherapy prototype. In 2001, the first clinical unit was completed and in 2002, the first human patient was treated at the University of Wisconsin. We will provide further details on some of the particularities of tomotherapy design, but in short, this treatment unit is basically a CT with an LINAC that replaces the x-ray tube.

The idea of a conventional multileaf collimator (MLC) used to modulate the field intensity was in the air in the 1990s, and other concepts were developed at the same time 


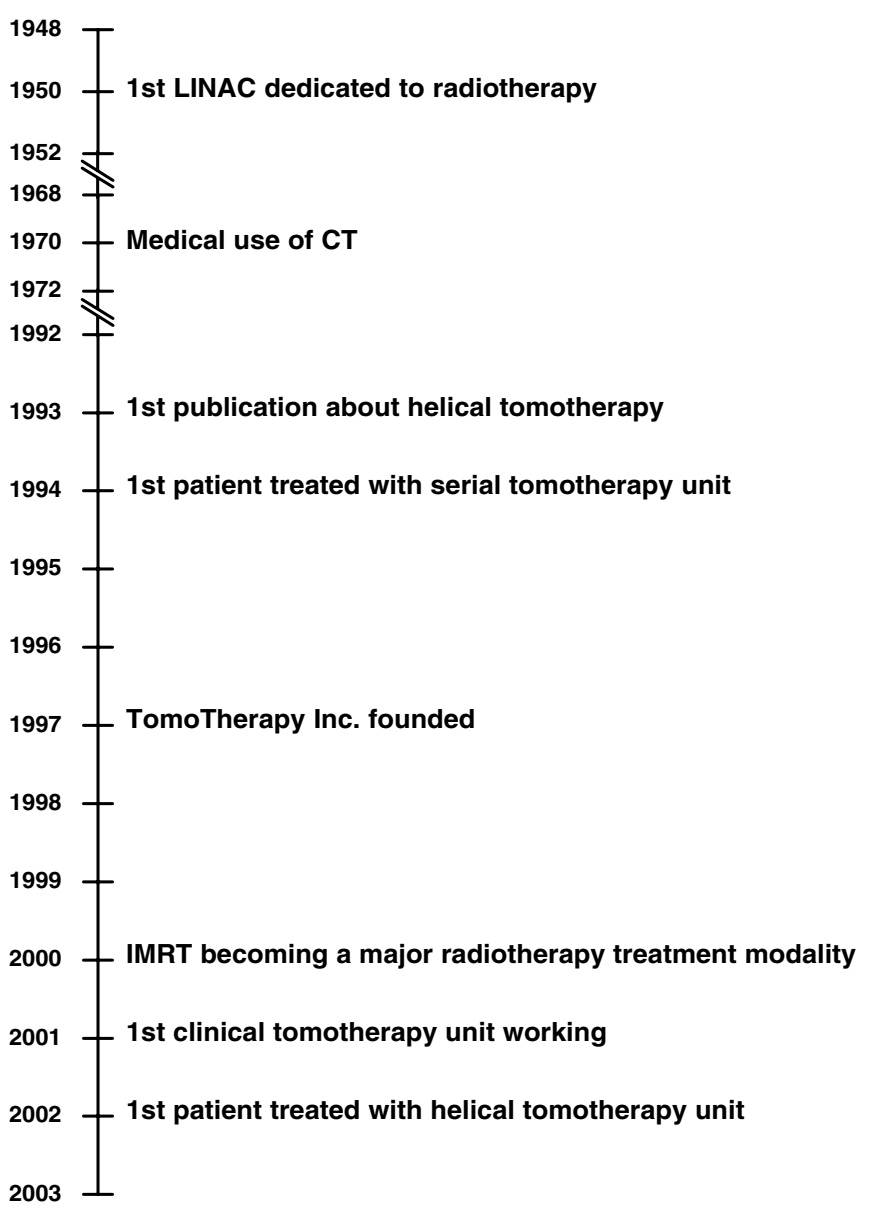

Figure 1. Major milestones leading to tomotherapy accelerators.

(Convery and Rosenbloom 1992, Carol 1995) which attained varying levels of commercial success. Carol's system was a success and the first patients were treated in 1994. The company changed its name to Nomos Corporation (Nomos Corporation, Swickley, PA, USA) and started selling the Peacock $^{\mathrm{TM}}$ in 1996. The treatment was described as serial tomotherapy, since it is delivered by a number of discrete arcs between which the treatment couch is moved. The Peacock ${ }^{\mathrm{TM}}$ uses an MLC, which can be added to an existing LINAC unit and clinics starting out with IMRT could upgrade instead of investing in a totally new accelerator.

The current commercial competitors for the title of the future of IMRT are based on the intensity-modulated arc therapy system (IMAT) first proposed by Yu (1995). The IMAT concept makes use of MLC-shaped fields, which define the intensity distribution at each angle (Yu 1995). In comparison with tomotherapy units, IMAT units do not deliver the dose using a slit or fan beam, which is fundamentally different because the dose is delivered in a single rotation. Another benefit is that IMAT relies on a concept applicable to conventional LINACs. However, IMAT did not achieve commercial success despite its promising potential, and later developments led to commercial volumetric modulated arc therapy (VMAT) units, which deliver the dose to the whole volume during a single gantry rotation (Otto 2008, Cao et al 2009, Webb and McQuaid 2009).
Current commercial units are the RapidArc ${ }^{\circledR}$ system (Varian Medical Systems Inc., Palo Alto, Ca, USA) and Elekta VMAT (Elekta AB, Stockholm, Sweden).

There are reports trying to quantify the respective advantages of each system; see Beavis (2004) and Korreman et al (2010) and, for more lively debates, see Bichay et al (2008), Ling et al (2008), Bortfeld and Webb (2009), Ling et al (2009), Mehta et al (2009), Mohan (2009), Otto (2009). Be aware that it is sometimes difficult to separate commercial, scientific and clinical arguments. Nowadays, technological advances do not always render previous techniques obsolete, and some competing solutions are still used simultaneously. The advantages of the different techniques and models become hard to quantify and it is not our place to seek epidemiological proofs of the supremacy of any particular technique. We refer the reader to better informed and more specialized publications, and we will stay out of the many debates running at the forefront of radiotherapy clinical results.

Some others in pursuit of the holy grail of radiotherapy chose a different technological path. In the past, neutron and ion radiotherapies were under development, but now, proton therapy is a leading candidate among emerging technologies. The localized energy deposition of protons could be advantageous to achieve highly conformal dose distributions and preserve healthy tissues (Brada et al 2007). Proton therapy still suffers from its very demanding infrastructure and is currently limited to a few specialized centres. Effort is currently being made to downsize the accelerator and thus the size of the gantry.

We will try in the following paragraphs to summarize the basic concepts of tomotherapy units as well as their implication for users and remaining challenges. We will describe some tomotherapy design particularities in comparison with conventional LINACs. Sections are also focused on quality assurance (QA), dosimetry procedures, radiation protection, dose distribution and treatment planning.

\section{Particularities of tomotherapy}

The following paragraphs are meant to explicate some of the particularities of tomotherapy. What we mean by particularity is a characteristic which differs from a conventional LINAC. We selected the particularities to discuss in an arbitrary fashion, and it is not our goal to be exhaustive or highly detailed.

\subsection{Design characteristics}

Tomotherapy is essentially the result of merging an LINAC and a CT in order to respond to the requirements of an ideal radiotherapy treatment. Figure 2 shows the general concept behind tomotherapy units. This particular heritage shows in some of its design characteristics, while others stem from choices of the development team.

The different components of the accelerator are visible on the picture of an opened unit, figure 3. Tomotherapy dose delivery to the patient is done helically, which is obtained by simultaneously moving the couch and the gantry, figure 2. A 64-leaf binary collimator modulates a fan-shaped field during 


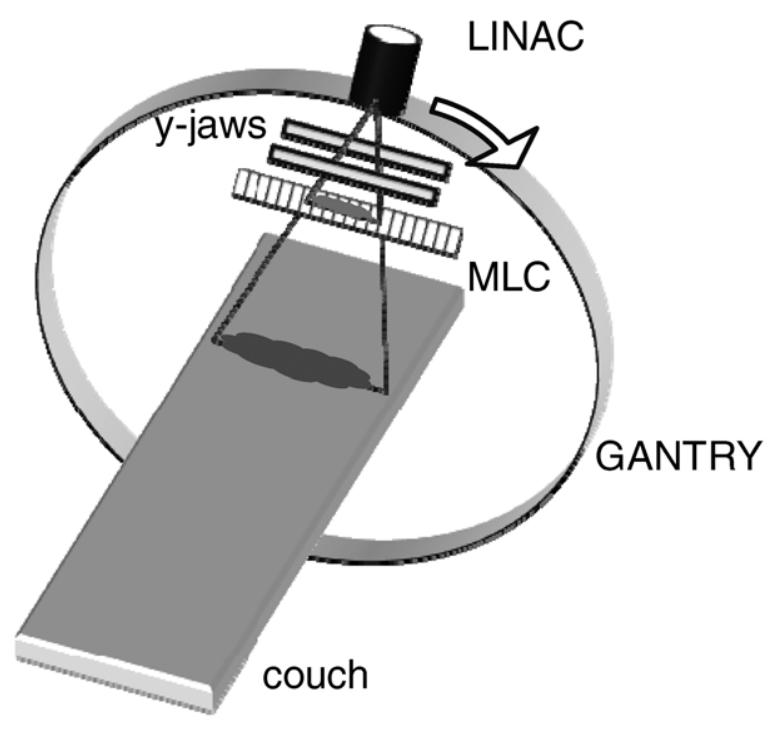

Figure 2. General principle of a tomotherapy unit.

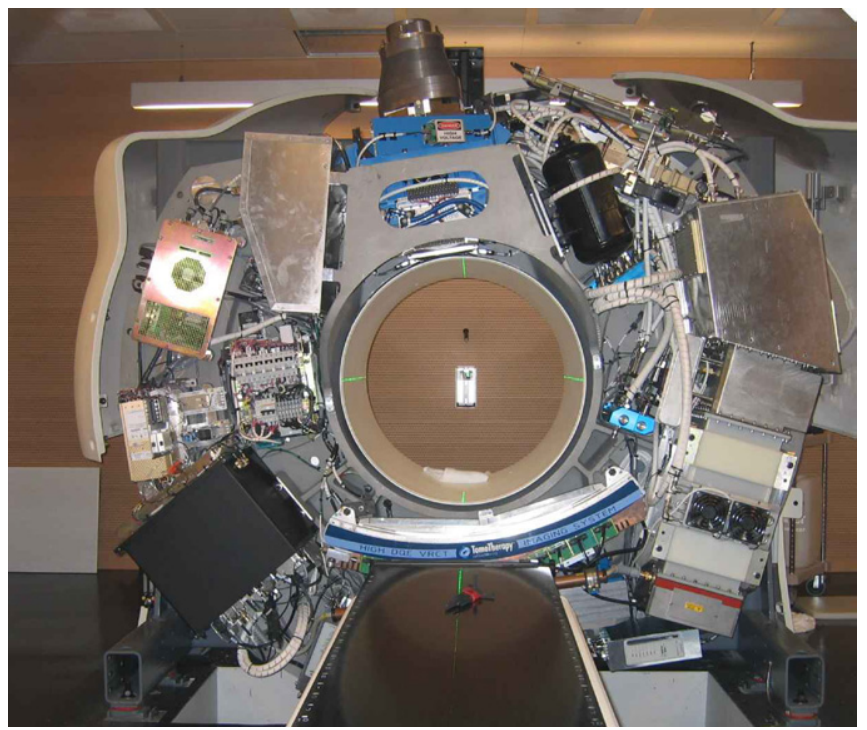

Figure 3. Open TomoTherapy Hi-Art II.

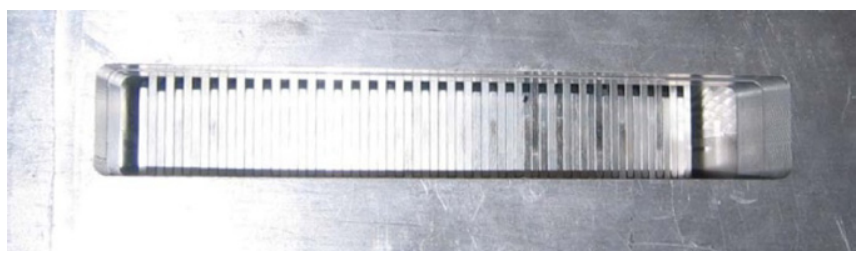

Figure 4. TomoTherapy Hi-Art II multileaf collimator.

delivery, figure 4 . The field's maximum transverse dimension is $40 \mathrm{~cm}$, and three fan beam widths are commissioned by the manufacturer, $1,2.5$ and $5 \mathrm{~cm}$. The beam width, defined by the $y$-jaw depicted in figure 2 , forbids the conventional reference beam size of $10 \mathrm{~cm} \times 10 \mathrm{~cm}$. The measure of the displacement speed of the couch in relationship to the gantry rotation speed and the field size is called the pitch and is an important parameter influencing the dose delivery.
Mackie et al (1993) described the ideas behind modern tomotherapy. A moving slip-ring gantry used for radiotherapy was introduced, which is essentially a standard CT gantry. The tomotherapy gantry was then adapted from a commercial HiSpeed gantry from General Electrics Medical (GEM), their first industrial partner. The inner diameter of the gantry limits the source-to-axis distance (SAD) at $85 \mathrm{~cm}$, instead of the usual $100 \mathrm{~cm}$ in conventional accelerators.

Another design particularity of tomotherapy is that its beam output is monitored in terms of absorbed dose per unit time. In a conventional accelerator, the beam intensity is monitored using units of dose per monitor unit. The tomotherapy output monitoring is achieved using two ion chambers located at the exit of the LINAC. The chambers check if the dose rate remains in a defined window. At the beginning of beam time, the output is known to be unstable, which is why the MLC stays closed for the initial $10 \mathrm{~s}$.

\subsection{Beam characteristics}

In LINACs as well as in x-ray tubes, incoming electrons produce bremsstrahlung in a high atomic number target. In a standard LINAC, the emitted photons go through a primary collimator, where the beam is shaped, and, then, through a flattening filter, where the beam fluence profile is flattened. Some of the physical properties of the emerging photons can be measured, but a complete description of the radiation field is only obtained using Monte Carlo (MC) calculations. Therefore, the description of tomotherapy beam characteristics will draw from measurements and MC results.

The complete characterization of a tomotherapy beam was first done by Jeraj et al (2004), and was later confirmed using beam model studies for dose verification (Caprile and Hartmann 2009, Sterpin et al 2010). It was reported that, despite its design, the tomotherapy energy spectrum did not significantly differ from a conventional LINAC with an incident electron energy of $6 \mathrm{MV}$.

A very important aspect of tomotherapy design is the absence of a flattening filter, which was already sketched in 1993 by Mackie et al (1993). The unit is a dedicated IMRT system and, therefore, does not need a flat dose profile. Consequently, the beam fluence profile presents as coneshaped, where the intensity in the centre is twice as large as on the sides, see figure 5. The lack of a flattening filter results in a higher dose rate, which reduces treatment duration. Higher dose rates can also be obtained in conventional LINACs by optimizing the accelerator, for example the Varian Trilogy (Varian Medical Systems Inc., Palo Alto, CA, USA). Additionally, the lack of a flattening filter helps reduce the head scatter radiation. Georg et al (2011) reported extensively on the perspective offered by flattening free filter units.

The flattening filter of a conventional LINAC has a strong contribution to the off-axis energy dependence of the beam. Consequently, only little decreases in the beam energy as a function of the angle are found. Jeraj et al (2004) reported only a $5 \%$ energy decrease between the centre and the edge of the tomotherapy beam, and the main effect was found above $3 \mathrm{MeV}$. 

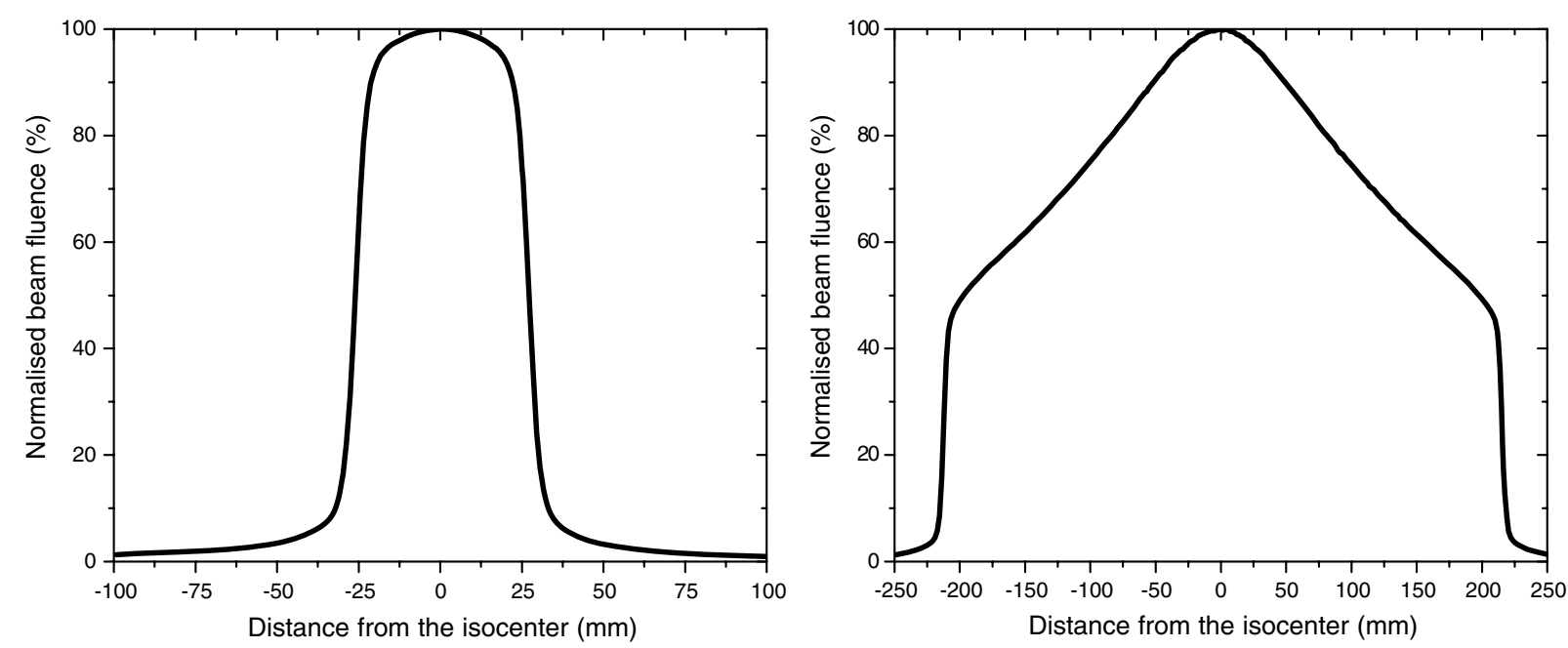

Figure 5. Typical beam fluence profiles in the longitudinal (left) and transverse (right) direction. The profiles were obtained experimentally using an ionization chamber, and the beam conditions were $5 \mathrm{~cm} \times 40 \mathrm{~cm}$.

The tomotherapy system has two clinical modes, treatment and imaging, which are obtained by varying the beam energy to $6 \mathrm{MV}$ and 3.5 MV, respectively. An additional physics mode is implemented as well, which is reserved for the unit warm up. The mean photon energy was found around $1.5 \mathrm{MeV}$ for the therapy mode and is reduced to $1.0 \mathrm{MeV}$ during imaging (Jeraj et al 2004). During imaging, the beam fluence is significantly reduced to preserve the patient. The unit output is consequently lowered by decreasing the incident electron energy, by reducing the pulse frequency and by narrowing the fan beam width to $4 \mathrm{~mm}$.

\subsection{Delivery characteristics}

MLCs have greatly evolved since their introduction as a replacement for shaping blocks. Nowadays, they are essential to providing conformal radiotherapy. Tomotherapy uses a 64-leaf binary MLC to modulate delivery field as fast as every $20 \mathrm{~ms}$, see figure 4 . It has a typical interlocking tongue-andgroove design in order to minimize leakage between leaves.

The beam is fan-shaped with a maximum transverse width of $40 \mathrm{~cm}$ and adjustable slice thickness of up to $5 \mathrm{~cm}$. The thickness is defined by the opening size of the secondary collimator (y-jaws in figure 2.), and a typical beam fluence profile in the longitudinal direction is shown in figure 5. It would even be possible to further increase the modulable components of a tomotherapy unit by including a dynamic secondary collimator (Mackie et al 1993, Gladwish et al 2004, Sterpin et al 2010, Sterzing et al 2010), which might be commercially available soon.

The configuration of a gantry that rotates around a moving couch results in a helical delivery pattern, which is common for CT, but unique in radiotherapy. A particular effect of the helical delivery arises at the overlap between fluence profiles of successive gantry laps. This effect has been studied by Kissick et al (2005) and was named the thread effect.

The thread effect refers to a particular fluence pattern noticeable on the delivery profile. It is a consequence of a poor choice of pitch. When the pitch is too high, the delivery profile of successive gantry laps will show some unevenness. Therefore, the pitch has to be carefully selected in order to avoid thread effects. Tomotherapy treatment parameters take this effect into account, and in clinical use, a pitch of 0.287 is recommended. No studies on the clinical relevance of the thread effect have yet been published.

A consequence of the helical mode is that the tomotherapy delivery superimposes offset fluence profiles on a helical trajectory. Any error along the couch axis will be repeated for each treatment slice and will be magnified. This reality necessitates maintaining strict QA requirements along the longitudinal axis (Balog et al 2003).

\subsection{Imaging characteristics}

Since their first use in the 1970s, CT imaging techniques in radiotherapy have become fairly standard. Historically, efforts to improve treatment accuracy were first aimed at creating better immobilization schemes and a generous coverage of the target volume. The introduction of imaging capabilities allowed radiotherapists to localize soft tissue structures more precisely, and on-board imaging systems opened a realm of new opportunities to optimize further delivery practices. Nobody would argue that CT is the standard for treatment planning, but on-board MVCT or CBCT provide adaptive planning opportunities. Inter-fraction uncertainties can be reduced, even when intra-fraction ones are still an issue for most technologies (Verellen et al 2008, Martin and Yartsev 2010). Various imaging systems have been tested on LINACs, for example electronic portal imaging devices (EPID), fluoroscopic imaging systems or ionization chambers, all of which can provide anatomical information to improve delivery accuracy (Keller et al 2002, Korreman et al 2010, Mackie et al 2003).

Tomotherapy has a built-in on-board MVCT, and, as previously explained, the LINAC beam fluence is decreased purposefully for imaging purposes. An advantage of such a configuration is that the imaging and treatment beams are inherently aligned, which eases any required quality 
controls. Tomotherapy imaging detector is a standard CT imaging system. An arc-shaped xenon detector is mounted on the gantry opposite to the LINAC. The detecting volume is composed of 640 submillimetre chambers separated by tungsten plates. Only 520 chambers are used for imaging, the standard image size is $512 \times 512$ pixels and the field of view is $40 \mathrm{~cm}$ wide (Mackie et al 2003).

In the frame of the QA of the reconstruction system, Keller et al (2002) studied the imaging characteristics of the detector. They found that the detector is suited for megavoltage CT imaging and image-guided radiotherapy, due to high efficiency and adequate resolution. The dose delivered for imaging purposes is consequently low, 10-30 mGy.

Another particularity of the mounting geometry is that the detector is seated at $129.2 \mathrm{~cm}$ from the LINAC's head but the focal point of the arc-shaped detector is at $103.6 \mathrm{~cm}$. This off-focus geometry was found to further increase the detector efficiency (Keller et al 2002).

\subsection{Treatment planning}

In radiotherapy, treatment planning involves the joint efforts of medical and physics experts to plan the appropriate geometric and radiological aspects of the therapy. Typically, a representation of the patient, obtained using imaging techniques, is used in a treatment planning system (TPS) to determine various treatment parameters. An extract of typical TPS dose distribution calculated for a tomotherapy unit is shown in figure 6. It is a typical head and neck tomotherapy treatment plan with concomitant boost. The prescribed doses are $70 \mathrm{~Gy}, 59.4 \mathrm{~Gy}$ and $52.8 \mathrm{~Gy}$ for three target volumes.

A characteristic of tomotherapy resides in its TPS, which uses an inverse planning dose calculation algorithm of convolution and superposition (Mackie et al 1985, 1988, Papanikolaou et al 1993). Briefly explained, the absorbed dose to water is obtained by convolution of the energy deposition kernel, which represents the relative energy deposited per volume unit, and the total energy released per unit of mass (TERMA).

The TPS calculates the delivered absorbed dose to water using data obtained with MC calculations. The full delivery is separated into single beamlets, which are defined as the potential radiation delivered by a single leaf in a single projection. They are calculated assuming 51 projections per rotation, spanning $7.06^{\circ}$ each, whereas the actual gantry rotation on the machine is continuous. Each projection has 64 beamlets, which are directly related to the 64 leaves of the MLC, and each beamlet is divided into multiple rays to ensure at least one ray per voxel. The calculation takes into account the specified field width, the dose calculation grid and the pitch. The pitch has to be smaller than 1 in order to cover each slice more than once. A smaller pitch allows more modulation, but induces longer delivery durations. Therefore, a compromise has to be found between the quality of the delivered dose, quantified by the modulation possibilities, and the delivery duration. As explained beforehand, the pitch must also be carefully chosen in order to minimize the thread effect.

The TERMA is calculated by averaging the primary rays which traverse the voxel volumes. The calculation uses a

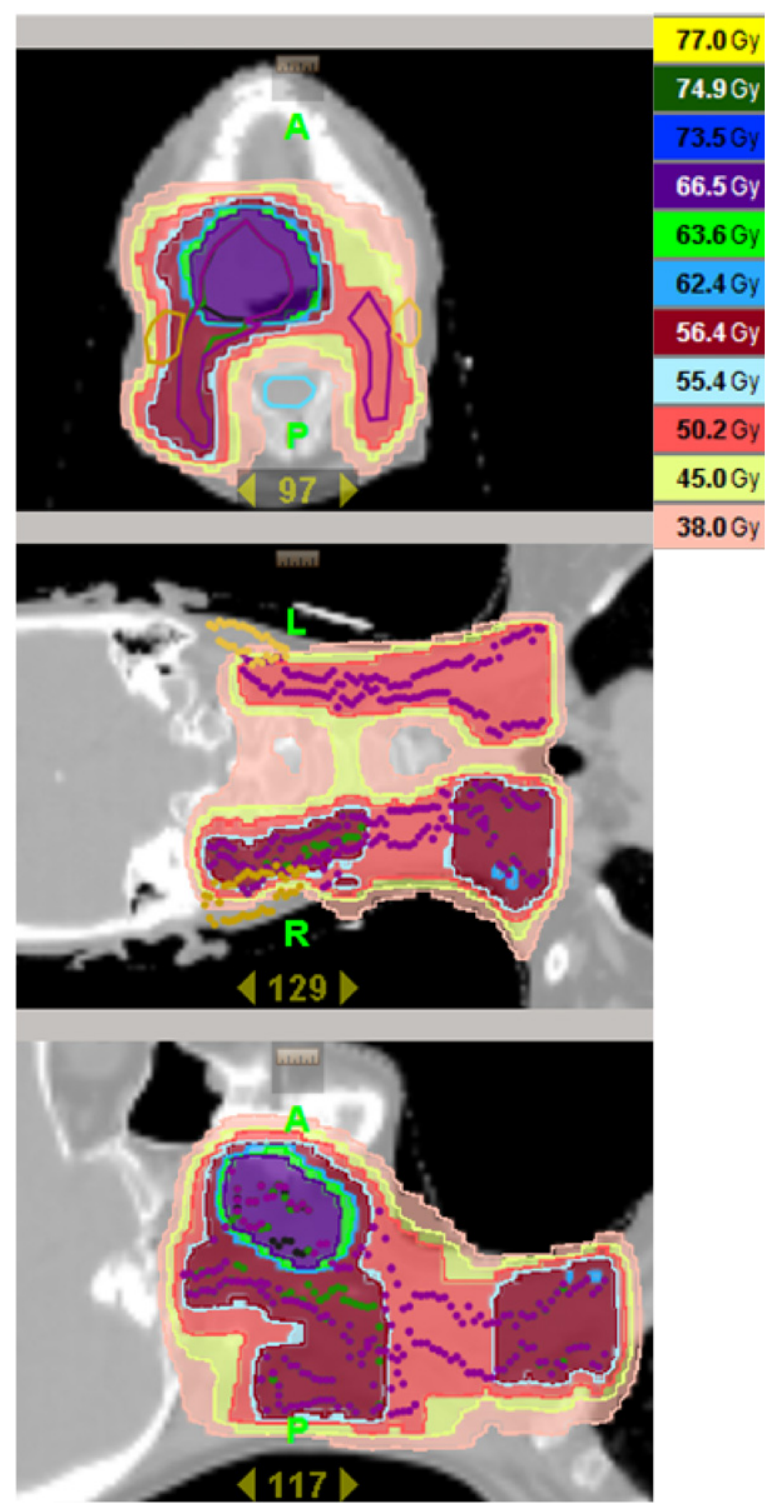

Figure 6. Extract of a typical head and neck treatment plan with concomitant boost. The prescribed doses are 70 Gy, 59.4 Gy and 52.8 Gy for three target volumes.

pre-computed fluence attenuation table (FAT), which gives attenuation coefficient values in water $\left(\mu_{\mathrm{w}} / \rho\right)$ and cortical bone $\left(\mu_{\mathrm{b}} / \rho\right)$ as a function of radiological depth. For intermediate densities, the attenuation coefficient is interpolated using the values of water and cortical bone. For densities lower than water, the calculations use the attenuation coefficient of water, and for densities greater than cortical bone, the attenuation coefficient of cortical bone is chosen. Beam hardening is taken into account using the FAT calculated using polyenergetic spectra. The spectral energy distribution is assumed constant across the beam, which is a reasonable assumption for accelerators without flattening filters (Jeraj et al 2004).

The polyenergetic deposition kernel is obtained by a weighted sum of monoenergetic kernels, based on the spectral energy distribution of the beam model. The convolution is then performed using the collapsed cone approximation (Ahnesjo 1989). 


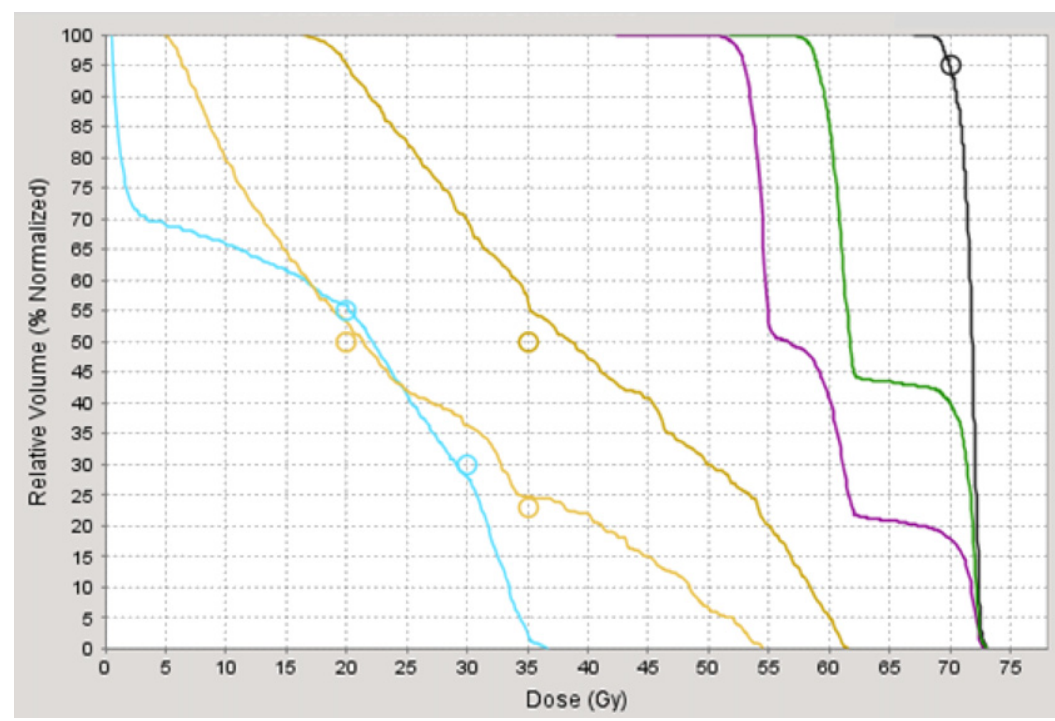

Figure 7. DVH of the treatment plan of figure 6. From left to right the curves are spinal cord, left parotid, right parotid, $52.8 \mathrm{~Gy}$ target volume, $59.4 \mathrm{~Gy}$ target volume, $70 \mathrm{~Gy}$ target volume.

The helical mode of treatment requires representing the MLC sequence for successive rotations, which is done using a sinogram. It shows the succession of opening times for each leaf at a given gantry angle during a given rotation. The initial sinogram is dimensioned to match the volume of the entire planning CT. An optimization of the sinogram according to the treatment goals is achieved by iterative leastsquares minimization, which was described in detail by Olivera et al (1998) and Shepard et al (2000). Another parameter of the calculations is the modulation factor, which is defined as the ratio between the maximum leaf open time and the average leaf open time. A higher modulation factor allows larger differences between respective beamlet weights, which induces a better dose volume histogram (DVH). A typical DVH obtained for a head and neck treatment is shown in figure 7.

However, the gantry speed needs to be low enough to match the longest beamlet opening time in the plan. After all optimization processes, full scatter calculations are performed taking into consideration the requirements of the fractionation schedule, the MLC, the couch and the gantry.

An interesting new approach to treatment parameter optimization has recently been developed. This approach is based on a non-voxel broad-beam (NVBB) framework (Lu 2010). The evaluation of the optimization process is generally done using an objective function, which quantifies how good the optimized solution is. The evaluation of the NVBB objective function is performed from a continuous point of view. Thus, pre-calculation and storage of beamlets are no longer needed. This framework can be implemented in a single graphic processing unit (GPU) due to low CPU requirements. This approach offers comparable dose accuracy, but with a strongly reduced planning time in comparison with the current TPS.

\subsection{Dose distribution}

Tomotherapy units are able to deliver high-quality conformal treatment, and compared with static modalities deliver lower doses to surrounding tissues, but these low doses are delivered over larger volumes. As already stated, there is no current consensus about the superiority of tomotherapy treatments compared with a dynamic IMRT treatment such as VMAT or RapidArc. There is abundant literature about treatment planning comparisons between the different solutions, for recent publication examples see Rao et al (2010), Marnitz et al (2010), Wiezorek et al (2011), Mavroidis et al (2011), Murthy et al (2011) or Zhou et al (2011), but the methodologies are sometimes hard to compare. Up to now, clinical evidence of the superiority of tomotherapy over other advanced treatment techniques has not been demonstrated in randomized trials or in prospective studies. Future clinical trials will have to compare delivering lower doses over a larger volume with larger doses over a smaller volume. This dose distribution raises concerns about out-of-field doses and risks of radiation-induced second cancers as well. It is our opinion that this matter should be resolved by clinicians and epidemiologists in the not too distant future.

\subsection{Commissioning and $Q A$}

Procedures for accepting a new radiotherapy accelerator are best conducted through the fruitful collaboration of the company and the local medical physicist. Many IAEA and AAPM documents review commissioning procedures for each separate component of the treatment delivery system. However, tomotherapy presents unique choices in terms of hardware and treatment design, which have implications on the initial commissioning process.

We will not illustrate the tomotherapy commissioning procedure in detail, which is mostly analogous to a conventional LINAC. Instead, we chose to emphasize two particularities.

Due to the calculation algorithm, which is based on convolution/superposition, the energy fluence spectrum and magnitude should be quantified during commissioning. Due 
to its helical delivery mode, the procedure is special for tomotherapy, and was described by Balog et al (2003).

Another tomotherapy particularity is that all planning systems use a common beam model, which is known as the gold standard, and is an average over the beam characteristics of the first ten tomotherapy units. This implies that each individual tomotherapy unit is adjusted to match the common beam model within $1 \%$. Therefore, it is essential to check that the commissioned tomotherapy unit still matches the common beam model implemented at the factory.

After collecting baseline data during the commissioning procedure, a QA program will ensure the constancy of the system. Early in the history of radiotherapy, QA systems were introduced. They are based on defined test protocols having a set frequency. QA systems are provided by recognized expert groups (for example the AAPM or the IAEA).

The complexity of the new technology has to be matched by the recommendations, which can result in a heavy work load for the medical physicists. Also, recommendations have a hard time keeping up with the rapidly evolving technology. However, it is essential that the culture of QA remains a priority within the radiotherapy community and, subsequently, the support for study and introduction of new protocols (Van Dyk 2008, Thwaites and Verellen 2010).

Before any published recommendations, many authors reported their QA procedures, some focusing more on specific tools and others with a precise description of their routine tomotherapy QA procedure (Fenwick et al 2004, 2005, Balog et al 2006, Broggi et al 2008, Cherpak et al 2008, Flynn 2008, Francois and Mazal 2009, Geurts et al 2009, Goddu et al 2009, Burnet et al 2010, Zhou et al 2010). A consensus exists on what to test but, sometimes, testing methods were different.

The AAPM recognized the challenges of tomotherapy, and the particularities of the system, which recently inspired them to make specific QA recommendations (Langen et al 2010). The broader objectives of the mandated AAPM task group were to propose QA procedures and dosimetry techniques. The report of Task Group 148 of the AAPM (Langen et al 2010) set QA requirements concerning delivery, imaging and treatment planning. Some noteworthy goals of the tomotherapy QA protocol are due to the complex mechanical design of the rotating LINAC, which necessitates checking the mechanical alignment of the LINAC with the primary collimator and with the MLC. Synchronizing the couch movement with the gantry is important, as well as verifying the gantry angles. Routine checks of static and helical beam output are recommended. Finally, the accuracy of the MVCT reconstructed images and of the image registration procedure should be tested.

\subsection{Radiation protection}

Report no. 151 of the National Council on Radiation Protection and Measurements (NCRP) describes guidelines for designing structural shielding of a radiotherapy facility using x-rays (NCRP 2005).

There are some specific issues related to room shielding calculations for tomotherapy. The rotation of the gantry makes the conventional usage factor obsolete, because it depends

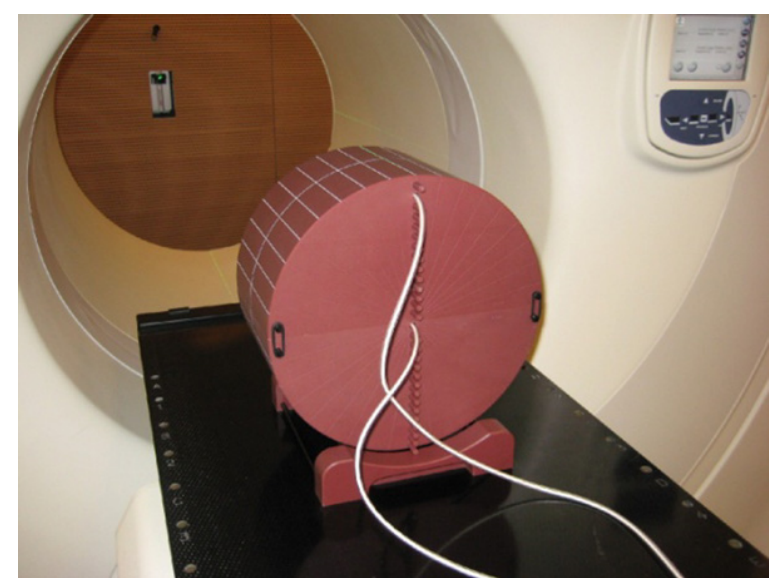

Figure 8. TomoTherapy Hi-Art II dosimetric system ready for use.

on beam direction, and tomotherapy is a dedicated IMRT, which significantly increases the workload factor. Shielding considerations for tomotherapy were already discussed in 2000 by Robinson et al (2000). This first paper could not go into much detail, because the unit design was not yet set, but the primary issues were already clearly identified. Practically, the LINAC should be carefully shielded first. Lead discs and tungsten blocks are used around the accelerator head. Moreover, the maximum longitudinal size of the beam is $5 \mathrm{~cm}$, which reduces the primary shielding, which is achieved using a $13 \mathrm{~cm}$ lead beam stop affixed to the gantry. Balog et al (2005) performed measurements around a tomotherapy unit and concluded that the scatter and primary radiations are negligible in comparison with the level of leakage radiation. They found the dose ratio of leakage to isocentre between $0.18 \%$ and $0.0006 \%$. They provided the reader with some typical calculations of the required wall thickness around the facility. Their results were later confirmed by other studies (Ramsey et al 2006, Kinhikar et al 2009). Practically, the recommendations by $\mathrm{Wu}$ et al (2006) on how to install a tomotherapy unit in an existing LINAC treatment room as well as the analytical model developed by Baechler et al (2007) may help future users to dimension their tomotherapy facility.

In conclusion, the AAPM generally recommends using a conventional accelerator bunker for the installation of a tomotherapy unit, but requires an extensive study in any case (Langen et al 2010).

\subsection{Dosimetry}

To proceed with commissioning, QA and dosimetry, tomotherapy units are delivered with a standard dosimetry system comprising some ionization chambers and a phantom. These tools are often cited in the literature concerning tomotherapy.

A cylindrical Virtual Water ${ }^{\mathrm{TM}}$ phantom (Gammex Inc., Middleton, WI, USA) is supplied, which is often referred to as the cheese phantom. It measures $30 \mathrm{~cm}$ diameter $\times 18 \mathrm{~cm}$ length of Virtual Water ${ }^{\mathrm{TM}}$, can be opened in half to use dosimetric films, and has lodgings for ionization chambers as well as reference density inserts, see figure 8. Virtual Water 


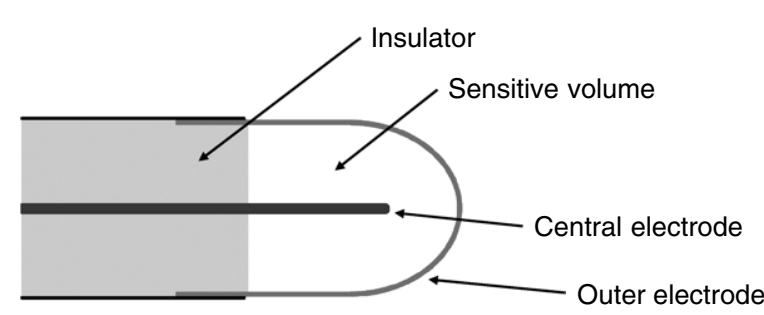

Figure 9. Basic design of an ionization chamber.

is a type of synthetic material, which mimics the attenuation and diffusion behaviour of water in a defined energy range. It is routinely used as an alternative to water tanks, which can be cumbersome to use.

Three types of chambers are provided as well. The reference ionization chamber is the Exradin A1SL ionization chamber (Standard Imaging, Middleton, WI, USA) with a collecting volume of $0.057 \mathrm{~cm}^{3}$. A Farmer-type chamber is available, the A12S (Standard Imaging, Middleton, WI, USA), but many users will already have a Farmer-type chamber on hand. A microchamber with a collecting volume of $0.016 \mathrm{~cm}^{3}$, the A14SL (Standard Imaging, Middleton, WI, USA), is sometimes provided for small field dosimetry. Finally a CT type chamber, the A17 (Standard Imaging, Middleton, WI, USA), with a collecting volume of $1.91 \mathrm{~cm}^{3}$, is used with the MVCT. The chambers are coupled to an electrometer called a Tomoelectrometer (Standard Imaging, Middleton, WI, USA). The calibration coefficients of the chambers are traceable to the American National Metrology Institute (NIST) in absorbed dose to water only for the beam quality of Co-60.

Figure 9 illustrates the basic design of ionization chambers. An ionization chamber's response depends on its geometry. Typically, the chamber sensitive volume can differ as well as its shape and size. The electrical properties of the electrometer, which measures the ionization current, is important as well. Gas-filled ionization chambers have been the gold standard in dosimetry until now. Recently, some practical limitations are becoming clear, for instance, the relatively large chamber size alters its response when measuring small fields, high dose gradients, and inhomogeneous fluence distributions. Of particular interest for small radiation fields is that the beam size is smaller than the range of secondary charged particles, which prevents charged particle equilibrium (CPE) from happening. CPE is a necessary condition for using cavity theory, which underlies measurements performed with ionization chambers. When CPE conditions are reached, the dose in a medium can be deduced from the dose in the air by applying a correction factor obtained by the stopping power ratios of the medium to air. In a small field, the presence of a detector can change the trajectories of the charged particles locally (Attix 1986, Gray 1929, 1936). Measuring time-dependent fluences is a challenge as well. MC calculations are recognized as a valuable tool, where measurements are not easily feasible. Using MC calculations validated experimentally, correction factors tailored for specific machines and detectors can be obtained and will be essential for the tomotherapy absolute dosimetry and treatment planning (Das et al 2008, Caprile and Hartmann 2009, Sterpin et al 2010).

Similarly to QA protocols, dosimetry recommendations are provided by recognized expert groups (AAPM or IAEA), but to this day, there is no such reference for the evaluation of the absolute dose of a tomotherapy unit. The need for a new international code of conduct for small radiation field radiotherapy is clear, because the conventional reference conditions are no longer adequate when applied to tomotherapy and other technologies, such as the RapidArc ${ }^{\circledR}$ system (Varian Medical Systems Inc., Palo Alto, CA, USA), VMAT and Leksell Gamma Knife® (Elekta AB, Stockholm, Sweden), Cyberknife ${ }^{\circledR}$ (Accuray Inc., Sunnyvale, CA, USA) and Vero Stereotactic Body Radiotherapy (SBRT) (Brainlab AG, Feldkirchen, Germany, and Mitsubishi Heavy Industries Ltd, Tokyo, Japan).

The absorbed dose output calibration of a conventional accelerator is described in the IAEA TRS-398 code of practice (Andreo et al 2000) and in the AAPM's TG-51 protocol (Almond et al 1999). The documents base an accelerator's absolute dosimetry on the use of an ion chamber, but mostly differ in the definition of the radiation beam quality. An adequate formalism for the dosimetry of small and composite fields was developed in order to extend the existing protocols (Alfonso et al 2008). Alfonso et al (2008) provide a framework for establishing a standard dosimetry applicable to a wide variety of technology. New parameters accounting for particular reference conditions as well as treatment modes were introduced. Some measurements and calculations tailored for tomotherapy were performed by Jeraj et al (2005), Thomas et al (2005), Xiong and Rogers (2008), Bailat et al (2009), Francois and Mazal (2009), Kragl et al (2009), Sauer (2009), Ceberg et al (2010), Palmans et al (2010). These studies can be used to obtain some of the parameters as well as correction factors to perform absolute dosimetry measurements.

The issue of beam size is not unique to tomotherapy, and manufacturers offer a variety of technical solutions. A nonexhaustive list comprises small volume ionization chambers, diode detectors, diamond detectors, MOSFET dosimeters, optically stimulated luminescent and thermoluminescent dosimeters, alanine dosimeters and films. The chosen technique will have to answer the requirements of the small field size as well as stringent metrological ones. However promising some technologies are, the weapon of choice remains the ionization chamber, and much effort is made to extend the methodology instead of changing technology.

Another challenge of tomotherapy is the helical treatment mode. The formalism introduced by Alfonso et al (2008) clearly recognizes the necessity of evaluating the helical dose delivery. The National Physical Laboratory in the UK (NPL) designed a methodology to provide reference dosimetry for Co-60, gamma-ray and megavoltage photon beams using alanine dosimeters (Duane et al 2006a, 2006b). This IMRT auditing system is mainly tailored for tomotherapy units. A Rexolite $^{\mathrm{TM}}$ phantom enables the simultaneous irradiation of alanine dosimeters and ionization chambers. Dosimetry using alanine is used routinely as a secondary standard by national metrology institutes. Alanine dosimeters are water-equivalent, 
can be located in a small volume, and show no strong energy dependence in the MV range. Alanine dosimeters coupled with electron spin resonance reading have uncertainties compatible with the requirement of absolute dosimetry procedures and are independent of many assumptions made to interpret ionization chamber results and provide an independent confirmation or chamber calibration (Anton 2006, Bailat et al 2009).

\section{Future opportunities}

Already in the original paper describing tomotherapy as well as in a review paper about IGRT (Mackie et al 1993, 2003), the development team identified potential opportunities for dose optimizations using MVCT data. Tomotherapy dose delivery is mathematically similar to tomographic imaging, and backprojection reconstruction algorithms can then be implemented for dose optimization. As a result, the obtained dosimetric data could be used in conjunction with a patient's treatment plan to tailor it for the next session.

The future usage of the MVCT data is central to several issues affecting dosimetric developments, but currently the MVCT data are only used for patient positioning. In the future, these images could be used to assess the actual dose delivered during a session knowing the patient's anatomy and to recalculate the treatment plan taking into account the patient anatomy of the day as well. The imaging system will have to be very stable in order to obtain usable MVCT images. Many studies have been published investigating the challenges and requirements of dose reconstruction using MVCT data (Kapatoes et al 2001a, 2001b, Langen et al 2005, Schombourg et al 2009, Duchateau et al 2010, Zhou et al 2010). In practice, the tomotherapy planning system uses Hounsfield units expressed in terms of mass density, instead of the conventional relative electron density. Therefore, in order to use MVCT images for dose evaluation, they have to be calibrated in terms of mass density. This calibration is performed using a cylindrical phantom with inserted reference density objects. A table of correspondence called an image value-to-density calibration table (IVDT) is then obtained. In order to use MVCT for daily patient dose evaluation, an IVDT will have to be obtained for each patient, which remains a practical problem.

Also, adaptive radiotherapy could be performed using a daily reconstructed dose distribution. The implementation of this technique still necessitates some studies as well as software development, and a commercial solution would be an exciting development. This new foreseen development will reduce inter-fraction uncertainties, but changes happening during the treatment fraction will remain a challenge (Verellen et al 2008, Martin and Yartsev 2010).

\section{Conclusions}

The ultimate cancer treatment would be able to kill all malignant tissues while preserving healthy ones. Currently, radiotherapy is one of the standard tools of radio oncologists, and it strives to reach the lowest toxicity possible. Since the time of the first medical LINACs in the 1950s, many developments worldwide made LINACs the radiotherapy instruments of choice over van de Graafs, betatrons and Co-60 irradiators. At the turn of the new millennium, radiotherapy gave birth to IMRT. The treatment zone is now defined by the intersection of numerous beamlets, which allows highly conformal irradiation volumes. A current IMRT system is the TomoTherapy ${ }^{\circledR} \mathrm{Hi}-\mathrm{Art}{ }^{\circledR}$ accelerators (Tomotherapy Inc., Madison, WI, USA), which began its life in the minds of scientists at the University of Wisconsin in the 1980s. Conceptually, the innovative idea behind tomotherapy units is to marry a CT unit with an LINAC. The obvious goal was to deliver beamlets from all radial directions around the patient and achieve a highly conformal dose distribution. Some competing IMRT units are IMATs, such as the RapidArc ${ }^{\circledR}$ system (Varian Medical Systems Inc., Palo Alto, CA, USA) and Elekta's VMAT (Elekta AB, Stockholm, Sweden).

The dose distribution delivered using a tomotherapy unit is indeed highly conformal, and compared with static modalities it delivers lower doses to surrounding tissues. However, the superiority of tomotherapy over other modalities remains to be clinically proven. Additionally, low doses are delivered over larger volumes compared with static modalities. Clinical studies will have to investigate the effects of delivering lower doses over larger volumes in comparison with delivering larger doses over smaller volumes.

Although the highly conformal treatment delivery is noteworthy, the use of on-board MVCT could lead to interesting progress as well. Effectively, images of a patient could be taken prior to the radiotherapy session and an evaluation of the treatment plan using the planning CT images could be performed. Furthermore, a reconstruction of the delivered dose could be performed afterwards and could enable the accurate correction of any patient inter-fraction variations. In the future, such technologies may allow simultaneous imaging and treatment, and therefore correct inter- and intrafraction variations.

In conclusion, TomoTherapy ${ }^{\circledR} \mathrm{Hi}-\mathrm{Art}^{\mathrm{TM}}$ is a promising system which meets some of the needs of the medical physicist community. Other manufacturers offer their own IMRT solution and the competition is fierce. The clinical results are still accumulating and epidemiological evaluations will help direct clinical choices in the future.

\section{References}

Ahnesjö A 1989 Collapsed cone convolution of radiant energy for photon dose calculation in heterogeneous media Med. Phys. 16 577-92

Alfonso R et al 2008 A new formalism for reference dosimetry of small and nonstandard fields Med. Phys. 35 5179-86

Almond P R, Biggs P J, Coursey B M, Hanson W F, Huq M S, Nath R and Rogers D W 1999 AAPM's TG-51 protocol for clinical reference dosimetry of high-energy photon and electron beams Med. Phys. 26 1847-70

Alongi F and Di Muzio N 2009 Image-guided radiation therapy: a new era for the radiation oncologist? Int. J. Clin. Oncol. 14 568-9

Andreo P, Burns D T, Hohlfeld K, Huq M S, Kanai T, Laitano F, Smythe V G and Vynckier S 2000 Absorbed dose determination in external beam radiotherapy: an international code of practice for dosimetry based on standards of absorbed 
dose to water IAEA Technical Report IAEA TRS-398 (Vienna: IAEA)

Anton M 2006 Uncertainties in alanine/ESR dosimetry at the Physikalisch-Technische Bundesanstalt Phys. Med. Biol. $515419-40$

Attix F H 1986 Introduction to Radiological Physics and Radiation Dosimetry (Weinheim: Wiley-VCH)

Baechler S, Bochud F O, Verellen D and Moeckli R 2007 Shielding requirements in helical tomotherapy Phys. Med. Biol. 52 5057-67

Bailat C J, Buchillier T, Pachoud M, Moeckli R and Bochud F O 2009 An absolute dose determination of helical tomotherapy accelerator, TomoTherapy High-Art II Med. Phys. 36 3891-6

Balog J, Olivera G and Kapatoes J 2003 Clinical helical tomotherapy commissioning dosimetry Med. Phys. 30 3097-106

Balog J, Lucas D, DeSouza C and Crilly R 2005 Helical tomotherapy radiation leakage and shielding considerations Med. Phys. 32 710-9

Balog J, Holmes T and Vaden R 2006 Helical tomotherapy dynamic quality assurance Med. Phys. 33 3939-50

Balter S and Balter J M 2008 Anniversary Paper: a sampling of novel technologies and the role of medical physicists in radiation oncology Med. Phys. 35 5641-52

Beavis A W 2004 Is tomotherapy the future of IMRT? Br. J. Radiol. 77 285-95

Bichay T, Cao D and Orton CG 2008 Helical tomotherapy will ultimately replace linear accelerator based IMRT as the best way to deliver conformal radiotherapy Med. Phys. 35 1625-8

Bortfeld T and Webb S 2009 Single arc IMRT? Phys. Med. Biol. 54 N9-N20

Brada M, Pijls-Johannesma M and De Ruysscher D 2007 Proton therapy in clinical practice: current clinical evidence J. Clin. Oncol. 25 965-70

Broggi S, Cattaneo G M, Molinelli S, Maggiulli E, Del Vecchio A, Longobardi B, Perna L, Fazio F and Calandrino R 2008 Results of a two-year quality control program for a helical tornotherapy unit Radiother. Oncol. 86 231-41

Burnet N G et al 2010 Practical aspects of implementation of helical tomotherapy for intensity-modulated and image-guided radiotherapy Clin. Oncol. 22 294-312

Cao D, Afghan M K, Ye J, Chen F and Shepard D M 2009 A generalized inverse planning tool for volumetric-modulated arctherapy Phys. Med. Biol. 54 6725-38

Caprile P and Hartmann G H 2009 Development and validation of a beam model applicable to small fields Phys. Med. Biol. 54 3257-68

Carol M P 1995 A system for planning and rotational delivery of intensity-modulated fields Int. J. Imag. Syst. Technol. 6 56-61

Ceberg C, Johnsson S, Lind M and Knöös T 2010 Prediction of stopping-power ratios in flattening-filter free beams Med. Phys. $371164-8$

Cherpak A, Studinski R C N and Cygler J E 2008 MOSFET detectors in quality assurance of tomotherapy treatments Radiother. Oncol. $86242-50$

Convery D J and Rosenbloom M E 1992 The generation of intensity-modulated fields for conformal radiotherapy by dynamic collimation Phys. Med. Biol. 37 1359-74

Das I J, Ding G X and Ahnesjö A 2008 Small fields: nonequilibrium radiation dosimetry Med. Phys. 35 206-15

Duane $\mathrm{S}$ et al 2006a Alanine and ion chamber dosimetry in helical tomotherapy Radiother. Oncol. 81 S45

Duane $\mathrm{S}$ et al 2006b Dosimetry audit for tomotherapy using alanine/EPR Med. Phys. 33 2093-4

Duchateau M, Tournel K, Verellen D, Van de Vondel I, Reynders T, Linthout N, Gevaert T, de Coninck P, Depuydt T and Storme G 2010 The effect of tomotherapy imaging beam output instabilities on dose calculation Phys. Med. Biol. 55 N329-N336
Fenwick J D et al 2004 Quality assurance of a helical tomotherapy machine Phys. Med. Biol. 49 2933-53

Fenwick J D, Tomé W A, Kissick M W and Mackie T R 2005 Modelling simple helically delivered dose distributions Phys. Med. Biol. 50 1505-17

Flynn R T, Kissick M W, Mehta M P, Olivera G H, Jeraj R and Mackie T R 2008 The impact of linac output variations on dose distributions in helical tomotherapy Phys. Med. Biol. $53417-30$

Francois P and Mazal A 2009 Static and rotational output variation of a tomotherapy unit Med. Phys. 36 816-20

Georg D, Knöös T and McClean B 2011 Current status and future perspective of flattening filter free photon beams Med. Phys. 38 1280-93

Geurts M, Gonzalez J and Serrano-Ojeda P 2009 Longitudinal study using a diode phantom for helical tomotherapy IMRT QA Med. Phys. 36 4977-83

Gladwish A, Kron T, McNiven A, Bauman G and Van Dyk J 2004 Asymmetric fan beams (AFB) for improvement of the craniocaudal dose distribution in helical tomotherapy delivery Med. Phys. 31 2443-8

Goddu S M, Mutic S, Pechenaya O L, Chaudhari S R, Garcia-Ramirez J, Rangaraj D, Klein E E, Yang D S, Grigsby J and Low D A 2009 Enhanced efficiency in helical tomotherapy quality assurance using a custom-designed water-equivalent phantom Phys. Med. Biol. 54 5663-74

Gray L H 1929 The absorption of penetrating radiation Proc. R. Soc. Lond. A 122 647-68

Gray L H 1936 An ionization method for the absolute measurement of $\gamma$-ray energy Proc. R. Soc. Lond. A 156 578-96

Hannoun-Levi J M, Lartigau E and Belkacemi Y 2010 From conventional radiation therapy to robotic approach: technological evolution and revolution of practice Oncologie 12 60-4

Huq S et al 2008 Transition from 2-D radiotherapy to 3-D conformal and intensity modulated radiotherapy IAEA-TECDOC-1588 (Vienna: IAEA)

Jeraj R, Mackie T R, Balog J, and Olivera G, Pearson D, Kapatoes J, Ruchala K and Reckwerdt P 2004 Radiation characteristics of helical tomotherapy Med. Phys. 31 396-404

Jeraj R, Mackie T R, Balog J and Olivera G 2005 Dose calibration of nonconventional treatment systems applied to helical tomotherapy Med. Phys. 32 570-7

Kapatoes J M, Olivera G H, Ruchala K J, Smilowitz J B, Reckwerdt P J and Mackie T R 2001a A feasible method for clinical delivery verification and dose reconstruction in tomotherapy Med. Phys. 28 528-42

Kapatoes J M, Olivera G H, Balog J P, Keller H, Reckwerdt P J and Mackie T R 2001b On the accuracy and effectiveness of dose reconstruction for tomotherapy Phys. Med. Biol. 46 943-66

Karzmark C J 1984 Advances in linear accelerator design for radiotherapy Med. Phys. 11 105-28

Keller H, Glass M, Hinderer R, Ruchala K, Jeraj R, Olivera G and Mackie T R 2002 Monte Carlo study of a highly efficient gas ionization detector for megavoltage imaging and image-guided radiotherapy Med. Phys. 29 165-75

Kinhikar R A, Jamema S V, Pai R, Sharmac P K D and Deshpande D D 2009 Radiation survey of first Hi-Art II Tomotherapy vault design in India Radiat. Meas. 44 188-92

Kissick M W, Fenwick J, James J A, Jeraj R, Kapatoes J M, Keller H, Mackie T R, Olivera G and Soisson E T 2005 The helical tomotherapy thread effect Med. Phys. 32 1414-23

Korreman S, Rasch C, McNair H, Verellen D, Oelfke U, Maingon P, Mijnheer B and Khoo V 2010 The European Society of Therapeutic Radiology and Oncology-European Institute of Radiotherapy (ESTRO-EIR) report on 3D CT-based in-room image guidance systems: a practical and technical review and guide Radiother. Oncol. 94 129-44 
Kragl G, af Wetterstedt S, Knäusl B, Lind M, McCavana P, Knöös T, McClean B and Georg D 2009 Dosimetric characteristics of 6 and 10 MV unflattened photon beams Radiother. Oncol. $93141-6$

Langen K M, Meeks S L, Poole D O, Wagner T H, Willoughby T R, Kupelian P A, Ruchala K J, Haimerl J and Olivera G H 2005 The use of megavoltage CT (MVCT) images for dose recomputations Phys. Med. Biol. 50 4259-76

Langen K M, Papanikolaou N, Balog J, Crilly R, Followill D, Goddu S M, Grant W, Olivera G, Ramsey C R and Shi C 2010 QA for helical tomotherapy: report of the AAPM Task Group 148 Med. Phys. 37 4817-53

Ling C C, Zhang P, Archambault Y, Bocanek J, Tang G and Losasso T 2008 Commissioning and quality assurance of RapidArc radiotherapy delivery system Int. J. Radiat. Oncol. Biol. Phys. 72 575-81

Ling C C, Archambault Y and Bocanek J 2009 Scylla and Charybis: longer beam-on time or lesser conformality-the dilemma of tomotherapy Int. J. Radiat. Oncol. Biol. Phys. 75 8-9

Lu W 2010 A non-voxel-based broad-beam (NVBB) framework for IMRT treatment planning Phys. Med. Biol. $557175-210$

Mackie T R, Scrimger J W and Battistag J J 1985 A convolution method of calculating dose for $15 \mathrm{MV}$ x-rays Med. Phys. 12 188-96

Mackie T R, Bielajew A, Rogers D W O and Battistag J J 1988 Generation of photon energy deposition kernels using the EGS Monte Carlo code Phys. Med. Biol. 33 1-20

Mackie T R, Holmes T, Swerdloff S, Reckwerdt P, Deasy J O, Yang J, Paliwal B and Kinsella T 1993 Tomotherapy: a new concept for the delivery of dynamic conformal radiotherapy Med. Phys. 20 1709-19

Mackie T R et al 2003 Image guidance for precise conformal radiotherapy Int. J. Radiat. Oncol. Biol. Phys. 56 89-105

Mackie T R 2006 History of tomotherapy Phys. Med. Biol. 51 R427-53

Marnitz S, Lukarski D, Köhler C, Wlodarczyk W, Ebert A, Budach V, Schneider A and Stromberger C 2010 Helical tomotherapy versus conventional intensity-modulated radiation therapy for primary chemoradiation in cervical cancer patients: an intraindividual comparison Int. J. Radiat. Oncol. Biol. Phys. at press (doi:10.1016/j.ijrobp.2010.06.005)

Martin S and Yartsev S 2010 kVCT, MVCT, and hybrid CT image studies - treatment planning and dose delivery equivalence on helical tomotherapy Med. Phys. 37 2847-54

Mavroidis P, Shi C, Plataniotis G A, Delichas M G, Ferreira B C, Rodriguez S, Lind B K and Papanikolaou N 2011 Comparison of the helical tomotherapy against the multileaf collimator-based intensity-modulated radiotherapy and 3D conformal radiation modalities in lung cancer radiotherapy Br. J. Radiol. 84 161-72

Mehta M, Hoban P and Mackie T R 2009 Commissioning and quality assurance of RapidArc radiotherapy delivery system: in regard to Ling et al (Int. J. Radiat. Oncol. Biol. Phys. 2008;72;575-581): absence of data does not constitute proof; the proof is in tasting the pudding Int. J. Radiat. Oncol. Biol. Phys. 75 4-6

Meredith W J 198440 years development in radiotherapy Phys. Med. Biol. 29 115-20

Mohan R 2009 Dueling technologies: in regard to Ling et al (Int. J. Radiat. Oncol. Biol. Phys. 2008;72: 575-81) Int. J. Radiat. Oncol. Biol. Phys. 75 6-8

Murthy V, Mallik S, Master Z, Sharma P K, Mahantshetty U and Shrivastava S K 2011 Does helical tomotherapy improve dose conformity and normal tissue sparing compared to conventional IMRT? A dosimetric comparison in high risk prostate cancer Technol. Cancer Res. Treatment 10 179-85
NCRP 2005 Structural shielding design and evaluation for megavoltage $\mathrm{x}$ - and gamma-ray radiotherapy facilities NCRP Report 151

Olivera G H, Shepard D M, Reckwerdt P J, Ruchala K, Zachman J, Fitchard E E, and Mackie T R 1998 Maximum likelihood as a common computational framework in tomotherapy Phys. Med. Biol. 43 3277-94

Otto K 2008 Volumetric modulated arc therapy: IMRT in a single gantry arc Med. Phys. 35 310-7

Otto K 2009 Letter to the Editor on 'Single-Arc IMRT?' Phys. Med. Biol. 54 L37

Palmans H, Thomas R A, Duane S, Sterpin E and Vynckier S 2010 Ion recombination for ionization chamber dosimetry in a helical tomotherapy unit Med. Phys. 37 2876-89

Papanikolaou N, Mackie T R, Meger-Wells C, Gehring M and Reckwerdt P 1993 Investigation of the convolution method for polyenergetic spectra Med. Phys. 20 1327-36

Ramsey C R, Seibert R, Mahan S L, Desai D and Chase D 2006 Out-of-field dosimetry measurements for helical tomotherapy system J. Appl. Clin. Med. Phys. 7 1-11

Rao M, Yang W, Chen F, Sheng K, Ye J, Mehta V, Shepard D and Cao D 2010 Comparison of Elekta VMAT with helical tomotherapy and fixed field IMRT: plan quality, delivery efficiency and accuracy Med. Phys. 37 1350-9

Robinson D, Scrimger J W, Field G C and Fallone B G 2000 Shielding considerations for tomotherapy Med. Phys. $272380-4$

Sauer O A 2009 Determination of the quality index (Q) for photon beams at arbitrary field sizes Med. Phys. 36 4168-72

Schombourg K, Bochud F and Moeckli R 2009 3D in vivo dosimetry in tomotherapy: first results Strahlentherapie Onkologie 185636

Shepard D M, Olivera G H, Reckwerdt P J and Mackie T R 2000 Iterative approaches to dose optimization in tomotherapy Phys. Med. Biol. 45 69-90

Sterpin E, Hundertmark B T, Mackie T R, Lu W G, Olivera G H and Vynckier S 2010 Monte Carlo-based analytical model for small and variable fields delivered by TomoTherapy Radiother. Oncol. 94 229-34

Sterzing F et al 2010 Dynamic jaws and dynamic couch in helical tomotherapy Int. J. Radiat. Oncol. Biol. Phys. 76 1266-73

Thomas S D, Mackenzie M, Rogers D W O and Fallone B G A 2005 Monte Carlo derived TG-51 equivalent calibration for helical tomotherapy Med. Phys. 32 1346-53

Thwaites D I and Tuohy J B 2006 Back to the future: the history and development of the clinical linear accelerator Phys. Med. Biol. 51 R343-R362

Thwaites D I and Verellen D 2010 Vorsprung durch Technik: evolution, implementation, QA and safety of new technology in radiotherapy Radiother. Oncol. 94 125-8

Van Dyk J 1999 The Modern Technology of Radiation Oncology: A Compendium for Medical Physicists and Radiation Oncologists (Madison, WI: Medical Physics Publishing Corporation)

Van Dyk J 2008 Quality assurance of radiation therapy planning systems: current status and remaining challenges Int. J. Radiat. Oncol. Biol. Phys. 71 S23-S27

Verellen D, De Ridder M and Storme G 2008 A (short) history of image-guided radiotherapy Radiother. Oncol. 86 4-13

Webb S and McQuaid D 2009 Some considerations concerning volume-modulated arc therapy: a stepping stone towards a general theory Phys. Med. Biol. 54 4345-60

Wiezorek T et al 2011 Rotational IMRT techniques compared to fixed gantry IMRT and tomotherapy: multi-institutional planning study for head-and-neck cases Radiat. Oncol. 6 20-30 
Wu C, Guo F and Purdy J 2006 Helical tomotherapy shielding calculation for an existing LINAC treatment room: sample calculation and cautions Phys. Med. Biol. 51 N389-N392

Xiong G M and Rogers D W O 2008 Relationship between $\% d d(10)_{x}$ and stopping-power ratios for flattening filter free accelerators: a Monte Carlo study Med. Phys. 35 2104-9

Yu CX 1995 Intensity-modulated arc therapy with dynamic multileaf collimation: an alternative to tomotherapy Phys. Med. Biol. 40 1435-49
Zhou J N, Uhl B, Dewit K, Young M, Taylor B, Fei D Y and Lo Y C 2010 Analysis of daily setup variation with tomotherapy megavoltage computed tomography Med. Dosim. 35 31-7

Zhou G X, Xu S P, Dai X K, Ju Z J, Gong H S, Xie C B, Yin L M and Yang J 2011 Clinical dosimetric study of three radiotherapy techniques for postoperative breast cancer: helical tomotherapy, IMRT, and 3D-CRT Technol. Cancer Res. Treatment 10 15-23 